\title{
Intravenous thrombolysis in Chinese patients with mild acute ischemic stroke
}

\author{
Yunyun Xiong ${ }^{1,2 \#}$, Ran Yan ${ }^{2 \#}$, Hongqiu Gu ${ }^{2,3}$, Shang Wang ${ }^{4}$, Marc Fisher $^{5}$, Xingquan Zhao ${ }^{1,2}$, Xin Yang ${ }^{1,3}$, \\ Chunjuan Wang ${ }^{1,3}$, Zhou Qi ${ }^{2}$, Xia Meng ${ }^{2}$, Zixiao Li ${ }^{1,2,3,6}$, Yongjun Wang ${ }^{1,2,3,6}$ \\ ${ }^{1}$ Vascular Neurology, Department of Neurology, Beijing Tiantan Hospital, Capital Medical University, Beijing, China; ${ }^{2}$ China National Clinical \\ Research Center for Neurological Diseases, Beijing, China; ${ }^{3}$ National Center for Healthcare Quality Management in Neurological Diseases, Beijing, \\ China; ${ }^{4}$ Neurocardiology Center, Department of Neurology, Beijing Tiantan Hospital, Capital Medical University, Beijing, China; ${ }^{5}$ Department of \\ Neurology, Stroke Division, Beth Israel Deaconess Medical Center, Harvard Medical School, Boston, MA, USA; ${ }^{6}$ Center for Stroke, Beijing Institute \\ for Brain Disorders, Beijing, China \\ Contributions: (I) Conception and design: Y Xiong, Z Li, Y Wang; (II) Administrative support: X Yang, X Meng; (III) Provision of study materials \\ or patients: X Zhao, C Wang; (IV) Collection and assembly of data: S Wang, X Yang, Z Qi, X Meng; (V) Data analysis and interpretation: H Gu, Y \\ Xiong, R Yan; (VI) Manuscript writing: All authors; (VII) Final approval of manuscript: All authors. \\ \#These authors contributed equally to the work. \\ Correspondence to: Yongjun Wang; Zixiao Li. China National Clinical Research Center for Neurological Diseases, Beijing, China; Vascular \\ Neurology, Department of Neurology, Beijing Tiantan Hospital, Capital Medical University, Beijing, China. Email: yongjunwang@ncrcnd.org.cn; \\ lizixiao2008@hotmail.com.
}

Background: Mild stroke accounts for more than a half of all stroke patients, and short-term outcomes after treatment with intravenous (IV) recombinant tissue plasminogen activator (rtPA) have not been fully investigated in this group.

Methods: Our study investigated short-term outcomes and predictors for a favorable functional outcome at discharge in mild stroke patients with IV rtPA. 6,752 mild stroke patients in the China Stroke Center Alliance with a clinical diagnosis of acute ischemic stroke, within 4.5 hours from symptom onset, with a baseline National Institutes of Health Stroke Scale score $\leq 5$ and received rt-PA treatment were included in this retrospective analysis. Univariable and multivariable analyses were performed to identify factors independently associated with a favorable functional outcome.

Results: Only $18.5 \%$ had an unfavorable functional outcome at discharge, $91.1 \%$ were discharged home, $89.9 \%$ could ambulate independently, $95.9 \%$ had a length of stay of 3 days or longer and $1.9 \%$ had sICH. A multivariable Logistic regression model identified that age $>80$ years [adjusted odds ratio (aOR): 1.57 (1.1-2.25)], diabetes mellitus [aOR: 1.35 (1.16-1.58)], 3-4.5 h time window [aOR: $1.43(1.26-1.63)$ ] and NIHSS score [3 vs. 0, aOR: 1.49 (1.05-2.11); 4 vs. 0, aOR: 2.36 (1.68-3.33); 5 vs. 0, aOR: 2.51 (1.77-3.56)] were independent risk factors for $\mathrm{mRS}>2$ with hospital region, hospital level and hypertension as covariates. Conclusions: Our findings suggest that tPA is safe and effective in mild stroke patients with age $\leq 80$ within the 3 hour time window and in those without diabetes mellitus, further studies are needed to confirm the findings.

Keywords: Thrombolysis; recombinant tissue plasminogen activator antigen (rtPA); stroke, ischemic; NIHSS; outcome

Submitted Jan 05, 2021. Accepted for publication Feb 26, 2021.

doi: 10.21037/atm-21-40

View this article at: http://dx.doi.org/10.21037/atm-21-40 


\section{Introduction}

In patients with ischemic stroke, mild strokes accounted for $\sim 50 \%$ (1), and these patients have a high risk of recurrence and consequently an increased risk of disability and death (2). Although intravenous (IV) thrombolysis using recombinant tissue plasminogen activator antigen (rtPA) is the firstline treatment for acute ischemic stroke within 4.5 hours of symptom onset and was recommended for patients with disabling mild stroke patients within 3 hours of symptom onset in the latest guidelines (3), only $13.5 \%$ of mild stroke patients received $\mathrm{rtPA}$ treatment (4).

A meta-analysis showed that IV rtPA could improve the odds of a favorable functional outcome for patients with a mild stroke within 4.5 hours of symptom onset (5). In addition, the Get With the Guidelines (GWTG) stroke registry reported that approximately $70 \%$ of mild stroke patients who received IV rtPA were able to return directly home or ambulate independently at discharge, and symptomatic intracranial hemorrhage (sICH) was infrequent (1.8\%) (4). However, the Potential of rtPA for Ischemic Strokes With Mild Symptoms (PRISMS) trial in patients with mild, nondisabling ischemic stroke revealed that rtPA was not superior to aspirin for a favorable functional outcome at 90 days (6). Nevertheless, the PRISMS trial was limited by premature termination and lacked enough power to make strong conclusions. Whether rtPA is effective for improving functional outcomes in patients with mild stroke is still controversial. Moreover, data on the effectiveness and safety of rtPA use in Asian patients with mild stroke are lacking.

Using a large scale prospective registry in China-the Chinese Stroke Center Alliance (CSCA) stroke registry (7), we evaluated short-term clinical outcomes and investigated the predictors for unfavorable functional outcome in mild stroke patients who have received IV rtPA within 4.5 hours of symptom onset. We present the following article in accordance with the STROBE reporting checklist (available at http://dx.doi.org/10.21037/atm-21-40).

\section{Methods}

The CSCA is a national, hospital-based, multicenter, voluntary, multifaceted intervention and continuous quality improvement initiative, as described elsewhere (7). The CSCA enrolled patients aged 18 years or older with a primary diagnosis of acute stroke or transient ischemic attack. The study was conducted in accordance with the Declaration of Helsinki (as revised in 2013). The study was approved by the Chinese Stroke Center Alliance (CSCA, the ethical reference number is KY 2018-06102). Participating hospitals received a healthcare quality assessment and research approval to collect data in the CSCA without requiring individual patient informed consent under the common rule or a waiver of authorization and exemption from their Institutional Review Board.

Data were collected via a web-based patient data collection and management tool (Medicine Innovation Research Center, Beijing, China), abstracted via chart review, coded, deidentified and transmitted securely to maintain patient confidentiality compliant with national privacy standards. We used a data abstraction tool which was characterized by predefined logic features, range checks and user alerts to identify a potentially invalid format or value entries and to optimize data quality at the time of entry. In addition, The China National Clinical Research Center for Neurological Diseases (NCRCND) served as the data analysis center and had an agreement to analyze the aggregate deidentified data for care quality feedback in order to improve the data quality.

\section{Subjects}

In our study, we included subjects who met the following inclusion criteria from the CSCA between March 2017 and August 2019 from 1,476 hospitals in China: (I) initial diagnosis of acute ischemic stroke confirmed by brain CT or MRI; (II) admission National Institutes of Health stroke scale (NIHSS) score $\leq 5$; (III) received IV $\mathrm{rtPA}$ within 4.5 hours from stroke onset. We excluded patients with a premorbid modified Rankin Scale (mRS) score $>1$, missing of major outcomes information or admission NIHSS scores.

An NIHSS score was assessed by neurologists certified in NIHSS evaluation at each hospital. We defined mild stroke as admission NIHSS score of $0-5(4,6,8)$. Baseline characteristics included demographics, patient management data (time from onset to arrival, door to needle time, symptom to needle time, first received care at Neurology Emergency and care in stroke unit), total dosage of rtPA, admission NIHSS score, medical history, medications prior to admission, in-hospital treatment, the hospitals' region (East China, Central China and Western China) and level (Tertiary or secondary hospital). 
Table 1 Patient characteristics

\begin{tabular}{|c|c|}
\hline Variables & Total $(\mathrm{N}=6,752)$ \\
\hline Age & $63.6 \pm 11.6$ \\
\hline \multicolumn{2}{|l|}{ Age group, years } \\
\hline$<45$ & $344(5.1)$ \\
\hline $45-65$ & $3,384(50.1)$ \\
\hline $66-80$ & $2,553(37.8)$ \\
\hline$>80$ & $471(7.0)$ \\
\hline Male & $4,606(68.2)$ \\
\hline $\begin{array}{l}\text { First received care at Neurology } \\
\text { Emergency }\end{array}$ & $2,233(33.1)$ \\
\hline Care in stroke unit & $2,344(34.7)$ \\
\hline Total dosage of rtPA & $55.0 \pm 11.7$ \\
\hline NIHSS at admission & $3.0(2.0-4.0)$ \\
\hline Hypertension & $4,014(59.4)$ \\
\hline Diabetes mellitus & $1,227(18.2)$ \\
\hline Dyslipidemia & $353(5.2)$ \\
\hline History of smoking & $2,717(40.2)$ \\
\hline Atrial fib/flutter & $378(5.6)$ \\
\hline Previous ischemic stroke or TIA & $1,281(19.0)$ \\
\hline Previous MI & $375(5.6)$ \\
\hline Carotid artery stenosis & $26(0.4)$ \\
\hline Prior antiplatelet use & $807(12.0)$ \\
\hline Prior anticoagulation use & $108(1.6)$ \\
\hline \multicolumn{2}{|l|}{ Hospital region } \\
\hline Eastern & $3,513(52.0)$ \\
\hline Central & $2,531(37.5)$ \\
\hline Western & $708(10.5)$ \\
\hline \multicolumn{2}{|l|}{ Hospital level } \\
\hline Secondary hospitals & $2,565(38.0)$ \\
\hline Tertiary hospitals & $4,187(62.0)$ \\
\hline \multicolumn{2}{|l|}{ Time measures } \\
\hline Time from onset to arrival, hours & $1.6(1.0-2.4)$ \\
\hline Symptom to needle time, hours & $2.7(2.0-3.4)$ \\
\hline Door to needle time, hours & $0.8(0.5-1.2)$ \\
\hline
\end{tabular}

rtPA, recombinant tissue plasminogen activator; TIA, transient ischemic attack; MI, myocardial infarction.

\section{Clinical outcomes}

An unfavorable functional outcome was defined as $\mathrm{mRS}>2$ at discharge, secondary outcomes included discharge home, independent ambulation at discharge $(\mathrm{mRS}<4)$ and length of stay ( $\geq 3 \mathrm{~d}$ ). The mRS scores were documented by local physicians, ranging from $0-6$, with 0 for no disability, 6 for death. Safety outcomes included sICH and gastrointestinal bleeding. sICH was defined as neurological worsening within 36 hours of rtPA administration with intracranial hemorrhage verified by computed tomography (CT) or magnetic resonance imaging (MRI), as documented by physicians.

\section{Statistical analysis}

To describe the clinical characteristics and clinical outcomes of rtPA in mild stroke, data are presented as number (percentage) for categorical variables and mean \pm SD for normally distributed continuous variables and median (interquartile range) for skewed data.

To determine the predictors for $\mathrm{mRS}>2$, univariable logistic regression with odds ratio (OR) [95\% confidence interval (CI)] are presented as a forest tree. Then, a multivariable logistic regression model with significant variables in the univariable logistic regression $(\mathrm{P}<0.05)$ was performed to identify the independent predictors for $\mathrm{mRS}>2$. Data were analyzed by SAS V.9.4 (SAS Institute Inc, Cary, NC), and \%ggBaseline, a SAS macro which can analyze and report baseline characteristics automatically (9), was used to generate tables for this study.

\section{Results}

Among 1,006,798 patients enrolled from 1,476 hospitals between March 2017 and August 2019, 16,507 patients with NIHSS $\leq 5$ received rtPA. After excluding 5,494 patients with missing data of clinical outcomes and 4,261 patients with premorbid mRS $>1,6,752$ patients were included in the analysis. The baseline characteristics of included subjects are described in Table 1 . The mean length of stay was $10.6 \pm 6.1$ days.

Table 2 shows short-term outcomes and treatment complications of rtPA in mild stroke patients. Among 6,752 patients analyzed, $18.5 \%$ had a mRS $>2$ at discharge, $89.9 \%$ had independent ambulation, 91.1\% were discharged home, 
and $95.9 \%$ had a length of stay of 3 days or longer. As for safety outcomes, no patient died, $1.9 \%$ had sICH and $0.6 \%$ had gastrointestinal bleeding.

The short-term outcomes among different NIHSS scores are summarized in Table 3. Overall, the higher the NIHSS score was, the higher the rates of unfavorable functional outcome was and the lower the rates of independent ambulation and discharge home were. sICH, gastrointestinal bleeding and length of stay were not statistically different across NIHSS scores.

Figure 1 shows the univariable analyses results for unfavorable functional outcome. NIHSS score, 3-4.5 hours window, age $>80$ years, hypertension, diabetes mellitus and receiving rtPA treatment in the secondary hospitals were associated with short-term unfavorable functional outcome. A multiple Logistic regression model found that age $>80$ years [adjusted OR (aOR) 1.57 (95\% CI: 1.1-2.25)],

Table 2 Short-term outcomes and treatment complications

\begin{tabular}{lc}
\hline Variables & Total $(\mathrm{N}=6,752)$ \\
\hline $\mathrm{mRS}>2$ & $1,250(18.5)$ \\
Independent ambulation at discharge & $6,073(89.9)$ \\
Discharge home & $6,148(91.1)$ \\
Length of stay $\geq 3 \mathrm{~d}$ & $6,474(95.9)$ \\
sICH & $130(1.9)$ \\
Gastrointestinal bleeding & $42(0.6)$ \\
\hline
\end{tabular}

*adjusted variables: total dosage of rtPA, first received care at neurology emergency, previous ischemic stroke or TIA, hospital region and hospital level. mRS, modified rankin scale; $\mathrm{sICH}$, symptomatic intracranial hemorrhage; OR, odds ratio; aOR, adjusted odds ratio; $\mathrm{Cl}$, confidence interval. diabetes mellitus [aOR 1.35 (95\%CI: 1.16-1.58)], 3-4.5 h time window [aOR 1.43 (95\% CI: 1.26-1.63)] and NIHSS score [3 vs. 0, aOR 1.49 (95\% CI: 1.05-2.11); 4 vs. 0, aOR 2.36 (95\% CI: 1.68-3.33); 5 vs. 0, aOR 2.51 (95\% CI: $1.77-$ 3.56)] were independently risk factors for $\mathrm{mRS}>2$ with hospital region, hospital level and hypertension as covariates (Table 4). Subgroup analysis in patients with NIHSS 0-1 revealed that old age $[>80$ vs. $<45$, aOR $6.38(95 \% \mathrm{CI}$ : 1.32-30.76)] and diabetes mellitus [aOR 1.6 (95\% CI: $1.02-$ 2.52)] were independently risk factors for $\mathrm{mRS}>2$ with time window as covariate.

\section{Discussion}

Our large prospective registry showed that favorable functional outcome was achieved by more than $80 \%$ of patients with infrequent sICH. Robust analyses revealed that 3-4.5 hour treatment window, age $>80$ years, diabetes mellitus and higher NIHSS score were risk factors for mRS $>2$ at discharge, similar to the overall stroke population.

Although several previous studies have reported the outcomes in mild stroke patients treated with rtPA, the power of these results were limited by a small sample size (10-12). The GWTG-Stroke Registry including 5,910 mild stroke patients yielded convincing results on the effectiveness and safety of rtPA (discharge home $70.6 \%$, independent ambulation $69.7 \%$, length of stay of 3 days or longer $73.0 \%$ and sICH $1.8 \%$ ) (4). Nevertheless, there is a paucity of evidence for the benefit of rtPA treatment in Chinese mild stroke patients. Our study is currently the largest sample investigating the effectiveness and safety of rtPA in patients with mild stroke. Patients with initial NIHSS 0-1 who received IV rtPA treatment may have

Table 3 Short-term outcomes and treatment complications among NIHSS Scores

\begin{tabular}{|c|c|c|c|c|c|c|c|}
\hline Variables & $\begin{array}{c}\text { NIHSS }=0 \\
(\mathrm{~N}=354)\end{array}$ & $\begin{array}{c}\text { NIHSS }=1 \\
(\mathrm{~N}=812)\end{array}$ & $\begin{array}{l}\mathrm{NIHSS}=2 \\
(\mathrm{~N}=1,408)\end{array}$ & $\begin{array}{l}\text { NIHSS }=3 \\
(\mathrm{~N}=1,466)\end{array}$ & $\begin{array}{l}\text { NIHSS }=4 \\
(\mathrm{~N}=1,549)\end{array}$ & $\begin{array}{l}\mathrm{NIHSS}=5 \\
(\mathrm{~N}=1,163)\end{array}$ & $P$ for trend \\
\hline$m R S>2$ & $42(11.9)$ & 75 (9.2) & $182(12.9)$ & $256(17.5)$ & $389(25.1)$ & $306(73.7)$ & $<0.001$ \\
\hline $\begin{array}{l}\text { Independent } \\
\text { ambulation at discharge }\end{array}$ & $334(94.4)$ & 776 (95.6) & 1,305 (92.7) & 1,333 (90.9) & $1,338(86.4)$ & 987 (84.9) & $<0.001$ \\
\hline Discharge home & $317(89.5)$ & $763(94.0)$ & $1,302(92.5)$ & 1,329 (90.7) & 1,393 (89.9) & 1,044 (89.8) & $<0.001$ \\
\hline $\mathrm{SICH}$ & $5(1.4)$ & $11(1.4)$ & $21(1.5)$ & $34(2.3)$ & $33(2.1)$ & $26(2.2)$ & 0.36 \\
\hline $\begin{array}{l}\text { Gastrointestinal } \\
\text { bleeding }\end{array}$ & $2(0.6)$ & $4(0.5)$ & $9(0.6)$ & $10(0.7)$ & $5(0.3)$ & $12(1.0)$ & 0.33 \\
\hline
\end{tabular}




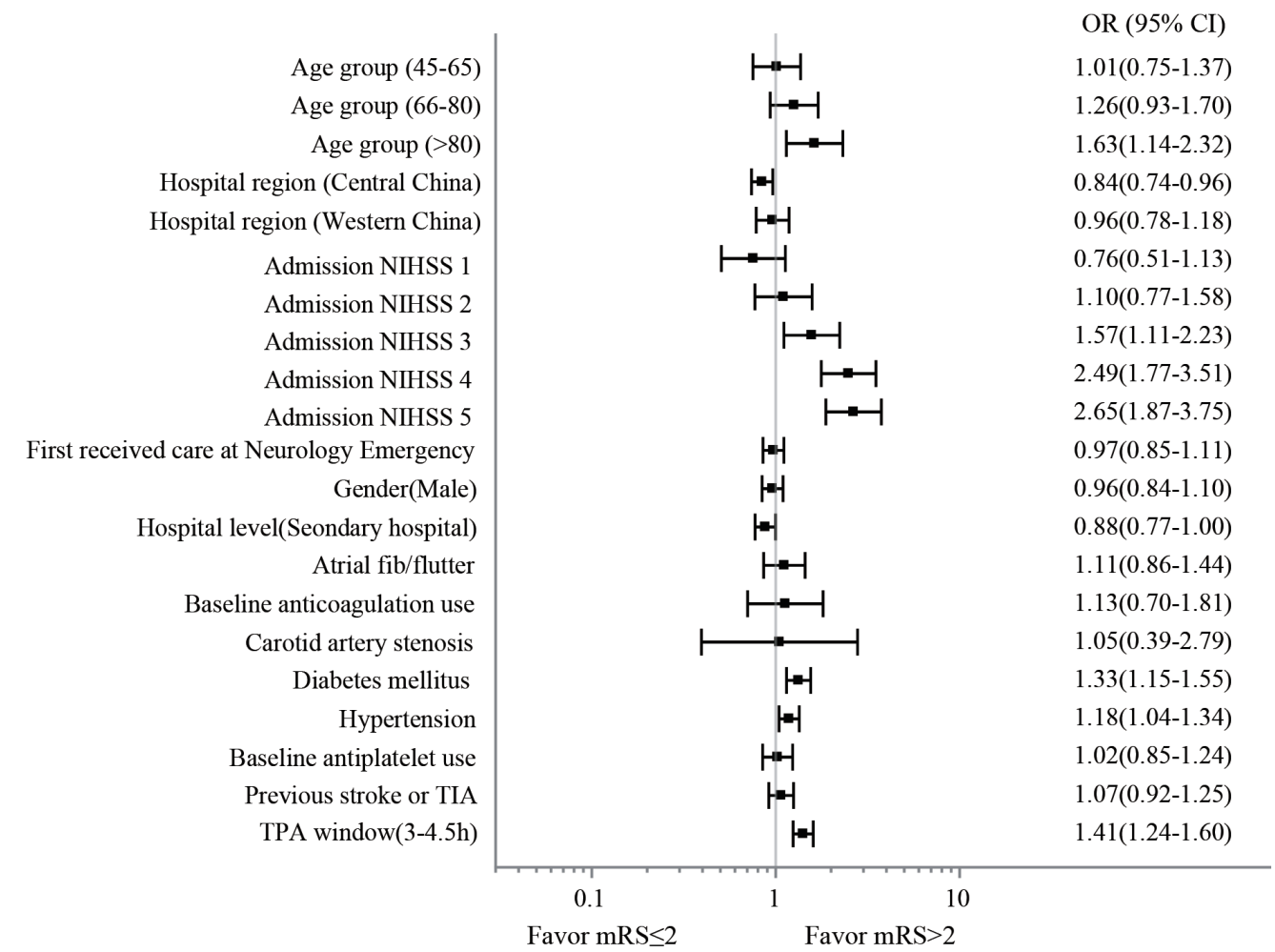

Figure $1 \mathrm{~A}$ forest tree to elucidate the univariate analysis for good functional outcome.

Table 4 Multiple Logistic regression result for $\mathrm{mRS}>2$ at discharge

\begin{tabular}{|c|c|c|}
\hline Variables & aOR (95\% Cl) & $P$ value \\
\hline $45-65$ vs. $18-45$ & $1(0.74-1.36)$ & 0.99 \\
\hline $66-80$ vs. $18-45$ & $1.22(0.9-1.65)$ & 0.20 \\
\hline$>80$ vs. $18-45$ & $1.57(1.1-2.25)$ & 0.01 \\
\hline 1 vs. 0 & $0.72(0.48-1.08)$ & 0.11 \\
\hline 2 vs. 0 & $1.04(0.73-1.49)$ & 0.84 \\
\hline 3 vs. 0 & $1.49(1.05-2.11)$ & 0.03 \\
\hline 4 vs. 0 & $2.36(1.68-3.33)$ & $<0.001$ \\
\hline $3-4.5$ vs. $0-3$ hours & $1.43(1.26-1.63)$ & $<0.001$ \\
\hline \multicolumn{3}{|l|}{ Diabetes mellitus } \\
\hline Yes vs. No & 1.35 (1.16-1.58) & $<0.001$ \\
\hline
\end{tabular}


been treated because their symptoms were fluctuating or because of the NIHSS score's inability to capture certain cerebellar and brainstem symptoms. In accordance with the GWTG-Stroke registry (4), we also found that the rate of sICH was quite low $(1.9 \%)$, which was significantly lower than the recently reported sICH rate in the PRISMS trial $(3.2 \%)(6)$ in mild stroke with NIHSS $0-5$ and previous rtPA clinical trials with higher NIHSS (5-6\%) (13-16). In addition, our sICH definition was similar with that in the GWTG-Stroke and the PRISMS trial. We defined sICH as neurological worsening within 36 hours of rtPA administration as documented by physicians. The definition of sICH is not identical to the National Institute of Neurological Disorders and Stroke criteria (13), it is similar and reasonably comparable. Consistent with the GWTGStroke study, sICH rates did not significantly differ among NIHSS scores. The consistent and robust low sICH rates in Chinese and Caucasians mild stroke populations suggest that the safety of rtPA is generalizable to a broad range of patients with mild stroke.

In comparison with the GWTG-Stroke study, we observed high rates of independent ambulation and discharge home, which may be due to the significantly longer length of stay in our study. In China, it usually takes 3-7 days for comprehensive examinations during hospitalization to be completed to better understand the mechanism of stroke, including carotid ultrasound, transcranial doppler with a bubble test, carotid CT angiography, MRI and MR angiography, 24-hour Holter, echocardiography, blood tests and if necessary highresolution MRI. Moreover, the rehabilitation team in stroke centers in China usually initiates bedside language and/or motor rehabilitation for hospitalized stroke patients within 2 days, which may be partly accounted for a higher rates of independent ambulation and directly discharge home as well.

We also observed that $81.5 \%$ of rtPA patients achieved a favorable functional outcome at discharge. Although this is slightly lower than the favorable functional outcome rate in the PRISMS trial (89.7\%), the follow-up duration of our study is significantly shorter than that in the PRISMS trial of 90 days (6). Consistent with previous studies, we also found that higher NIHSS score, 3-4.5 hours window and diabetes mellitus are robust and important risk factors for unfavorable functional outcome at discharge (4,5,17-20).

Our study provides insight on the effectiveness and safety of rtPA in mild stroke based on a large nationally representative prospective registry. It supports rtPA treatment in Asian patients with mild acute ischemic stroke.
There are limitations to our study. First, we did not have information on patients' NIHSS subitems (consciousness, aphasia, hemianopsia, motor, etc.), which could not allow us to differentiate whether the mild stroke was disabling or non-disabling. Further studies are warranted to investigate the efficacy and safety of rtPA in disabling mild stroke. Second, our study is a prospective registry, which may have selection bias and can not substitute for a randomized clinical trial to demonstrate the efficacy of rtPA in mild stroke. Although the PRISMS trial did not prove that rtPA was more beneficial than aspirin in non-disabling mild stroke, early termination with less than the anticipated number of patients caused a lack of power to make firm conclusions. Further clinical trials in mild stroke comparing rtPA with aspirin or aspirin plus clopidogrel are needed to provide conclusive evidence of rtPA benefit in mild stroke patients with longer-term outcomes. Third, more than 50\% of minor stroke patients in the registry were excluded due to lack of data or mRS $>1$ prestroke, that might have caused bias in the analysis. Fourth, we lacked information regarding stroke localization and etiology, which are important confounders for functional outcome. Lastly, patients with large vessel occlusion presenting as a mild stroke at admission may have stroke progression and unfavorable functional outcome at discharge, however, we lacked information of large vessel occlusion. Considering that $2.7 \%$ mild stroke patients had large vessel occlusion (21), the findings in the current study may not be affected by the lack of information on large vessel occlusion.

\section{Conclusions}

Our findings suggest that rtPA is safe and effective in mild stroke patients with an age $\leq 80$, within 3 hours time widow, and in those without diabetes mellitus. Our observation provides more evidence that tPA may be useful in mild stroke patients, but further studies are needed to confirm the findings.

\section{Acknowledgments}

The language of this paper was edited by Dr. Marc Fisher. Funding: This work was supported by grants from the National Key R\&D Program of China (2017YFC1310901, 2018YFC1312903), Beijing Municipal Science \& Technology Commission (D171100003017002), National Natural Science Foundation of China (81671172), Beijing Talents Project (2018A13), and National Science and 
Technology Major Project (2017ZX09304018).

\section{Footnote}

Reporting Checklist: The authors have completed the STROBE reporting checklist. Available at http://dx.doi. org/10.21037/atm-21-40

Peer Review File: Available at http://dx.doi.org/10.21037/ atm-21-40

Conflict of Interest: All authors have completed the ICMJE uniform disclosure form (available at http://dx.doi. org/10.21037/atm-21-40). The authors have no conflicts of interest to declare.

Ethical Statement: The authors are accountable for all aspects of the work in ensuring that questions related to the accuracy or integrity of any part of the work are appropriately investigated and resolved. The study was conducted in accordance with the Declaration of Helsinki (as revised in 2013). The study was approved by the Chinese Stroke Center Alliance (CSCA, the ethical reference number is KY 2018-061-02). Participating hospitals received a healthcare quality assessment and research approval to collect data in the CSCA without requiring individual patient informed consent under the common rule or a waiver of authorization and exemption from their Institutional Review Board.

Open Access Statement: This is an Open Access article distributed in accordance with the Creative Commons Attribution-NonCommercial-NoDerivs 4.0 International License (CC BY-NC-ND 4.0), which permits the noncommercial replication and distribution of the article with the strict proviso that no changes or edits are made and the original work is properly cited (including links to both the formal publication through the relevant DOI and the license). See: https://creativecommons.org/licenses/by-nc-nd/4.0/.

\section{References}

1. Wang Y, Li Z, Wang Y, et al. Chinese stroke center alliance: A national effort to improve healthcare quality for acute stroke and transient ischaemic attack: Rationale, design and preliminary findings. Stroke Vasc Neurol 2018;3:256-62.

2. Reeves M, Khoury J, Alwell K, et al. Distribution of national institutes of health stroke scale in the cincinnati/ northern kentucky stroke study. Stroke 2013;44:3211-3.

3. Powers WJ, Rabinstein AA, Ackerson T, et al. Guidelines for the early management of patients with acute ischemic stroke: 2019 update to the 2018 guidelines for the early management of acute ischemic stroke: A guideline for healthcare professionals from the american heart association/american stroke association. Stroke 2019;50:e344-e418.

4. Romano JG, Smith EE, Liang L, et al. Outcomes in mild acute ischemic stroke treated with intravenous thrombolysis: A retrospective analysis of the get with the guidelines-stroke registry. JAMA Neurol 2015;72:423-431.

5. Emberson J, Lees KR, Lyden P, et al. Effect of treatment delay, age, and stroke severity on the effects of intravenous thrombolysis with alteplase for acute ischaemic stroke: A meta-analysis of individual patient data from randomised trials. Lancet 2014;384:1929-35.

6. Khatri P, Kleindorfer DO, Devlin T, et al. Effect of alteplase vs aspirin on functional outcome for patients with acute ischemic stroke and minor nondisabling neurologic deficits: The prisms randomized clinical trial. JAMA 2018;320:156-66.

7. Duan Z, Wang H, Wang Z, et al. Neutrophil-lymphocyte ratio predicts functional and safety outcomes after endovascular treatment for acute ischemic stroke. Cerebrovasc Dis 2018;45:221-7.

8. Khatri P, Tayama D, Cohen G, et al. Effect of intravenous recombinant tissue-type plasminogen activator in patients with mild stroke in the third international stroke trial-3: Post hoc analysis. Stroke 2015;46:2325-7.

9. Gu HQ, Li DJ, Liu C, Rao ZZ. \%ggbaseline: A sas macro for analyzing and reporting baseline characteristics automatically in medical research. Ann Transl Med 2018;6:326.

10. Khatri P, Kleindorfer DO, Yeatts SD, et al. Strokes with minor symptoms: An exploratory analysis of the national institute of neurological disorders and stroke recombinant tissue plasminogen activator trials. Stroke 2010;41:2581-6.

11. I. S. T. collaborative group. Effect of thrombolysis with alteplase within $6 \mathrm{~h}$ of acute ischaemic stroke on longterm outcomes (the third international stroke trial [ist3)]: 18-month follow-up of a randomised controlled trial. Lancet Neurol 2013;12:768-776.

12. Kohrmann M, Nowe T, Huttner HB, et al. Safety and outcome after thrombolysis in stroke patients with mild symptoms. Cerebrovasc Dis 2009;27:160-6.

13. National Institute of Neurological Disorders and Stroke 
rt-PA Stroke Study Group. Tissue plasminogen activator for acute ischemic stroke. N Engl J Med 1995;333:1581-7.

14. Hacke W, Donnan G, Fieschi C, et al. Association of outcome with early stroke treatment: Pooled analysis of atlantis, ecass, and ninds rt-pa stroke trials. Lancet 2004;363:768-774.

15. Hacke W, Kaste M, Bluhmki E, et al. Thrombolysis with alteplase 3 to 4.5 hours after acute ischemic stroke. N Engl J Med 2008;359:1317-29.

16. Clark WM, Wissman S, Albers GW, et al. Recombinant tissue-type plasminogen activator (alteplase) for ischemic stroke 3 to 5 hours after symptom onset. The atlantis study: A randomized controlled trial. Alteplase thrombolysis for acute noninterventional therapy in ischemic stroke. JAMA 1999;282:2019-26.

17. Kamal N, Sheng S, Xian Y, et al. Delays in door-to-needle

Cite this article as: Xiong Y, Yan R, Gu H, Wang S, Fisher M, Zhao X, Yang X, Wang C, Qi Z, Meng X, Li Z, Wang Y. Intravenous thrombolysis in Chinese patients with mild acute ischemic stroke. Ann Transl Med 2021;9(9):767. doi: 10.21037/atm-21-40 times and their impact on treatment time and outcomes in get with the guidelines-stroke. Stroke 2017;48:946-54.

18. Caso V, Paciaroni M, Venti M, et al. Determinants of outcome in patients eligible for thrombolysis for ischemic stroke. Vasc Health Risk Manag 2007;3:749-54.

19. Wouters A, Nysten C, Thijs V, et al. Prediction of outcome in patients with acute ischemic stroke based on initial severity and improvement in the first $24 \mathrm{~h}$. Front Neurol 2018;9:308.

20. Desilles JP, Meseguer E, Labreuche J, et al. Diabetes mellitus, admission glucose, and outcomes after stroke thrombolysis: A registry and systematic review. Stroke 2013;44:1915-23.

21. Manno C, Disanto G, Bianco G, et al. Outcome of endovascular therapy in stroke with large vessel occlusion and mild symptoms. Neurology 2019;93:e1618-26. 